\title{
A Ferramenta "Tabulação SIC" e sua influência na Ação Projetual e na Gestão de Sistemas Complexos
}

\section{The SIC Index Tool and its influence in the design and management of complex systems}

\author{
${ }^{1}$ Marcello Silva e Santos \\ ${ }^{2}$ Alessandra Reis Nascimento
}

1 Docente do Curso de Engenharia de Produção do Centro Universitário de Volta Redonda - UniFOA / marcellosanto@hotmail.com
2 Graduada em Engenharia de Produção - UniFOA / alessandra.rnascimento@yahoo.com.br

\section{RESUMO}

Projetos de salas de controle de refinarias envolvem a combinação de grande número de variáveis e especificidades que fazem com que os mesmos sejam complexos, não apenas em sua natureza como no seu tratamento. Em outras palavras, deve-se trabalhar a ação projetual de forma integrada, associando especificações técnicas, exigências de segurança e ambientais, além de necessidades particulares da organização do trabalho nesse tipo de ambiente construído. A ergonomia, portanto, aqui entendida como a disciplina científica que busca adaptar os sistemas de trabalho às necessidades humanas, é um importante componente dessa equação, agregando qualidade ao projeto. Entretanto, necessidades, prioridades e requisitos operacionais devem ser ponderados cuidadosamente em função dos usuários. Este artigo aborda a utilização de uma ferramenta de priorização, desenvolvida em conjunto pelo UniFOA e o Laboratório GENTE, da COPPE/UFRJ. A Tabulação SIC é uma ferramenta que auxilia e priorização dos requisitos de ergonomia na definição das anotações de projeto que devem ser incorporadas no projeto executivo.

\section{PALAVRAS-CHAVE}

Sistemas complexos; ergonomia; organização do trabalho.

\begin{abstract}
The design of Oil Refinery Control Rooms combines several variables and technical details, making project management a complex challenge. Thus, integration is key in order to accomplish such demand,binding together tech specs, safety requirements, environmental guidelines and, particularly, job design constraints that prevail in those workspaces. Ergonomics, understood herein as a scientific discipline that seeks to adapt work systems to human needs, is an important component in complex systems design projects. However,needs, priorities and operational requirements varyfrom worker to worker, so actions must be properly balanced to yield to consensual solutions for different stakeholders. This paper deals with the application of a prioritizing tool, which tallies and combines multiple variables related to work conditions, task frequency, occupational severity and so on. The SIC tool produces inputs for design improvement, because it incorporates into project plans real world experiences, impossible to retrieve in a non-materialized workspace. Initial results show that the tool serves its purpose not only for ergonomic work analysis and project management processes, but also in terms of proving valuabe design guidelines.
\end{abstract}

\section{KEYWORDS}

Complex Systems; ergonomic design; job design.

\section{Como você deve citar?}

SILVA E SANTOS, Marcello; NASCIMENTO, Alessandra Reis. A Ferramenta "Tabulação SIC" e sua influência na Ação Projetual e na Gestão de Sistemas Complexos. Cadernos UniFOA, Volta Redonda, $n^{\circ} 25$, quadrimestral, p. 7-18, ago. 2014. 


\section{INTRODUÇÃO}

Este artigo trata das ações relacionadas a um PIC - Projeto de Iniciação Científica, desenvolvido a partir de uma análise ergonômica realizada no $\mathrm{CCl}$ (Centro de Controle Integrado) de uma refinaria de petróleo. As ações de apoio à pesquisa fazem parte do escopo de um contrato de consultoria técnica celebrado entre a refinaria e o Laboratório GENTE/COPPE, vinculado à UFRJ - Universidade Federal do Rio de janeiro, através da Fundação COPPETEC. A Fundação dá suporte de gestão aos contratos entre aquela instituição federal e o mercado como um todo. Em resumo, as ações nesse PIC estão limitadas à tabulação dos dados obtidos pelos pesquisadores do laboratório. As seguintes etapas foram cumpridas no contexto global do projeto:

a). Coletas de dados e análises observacionais e conversacionais, que serão apresentados em forma de relatório, segundo o modelo do método SPM ${ }^{\circledR}$, desenvolvido pela equipe de pesquisadores laboratório;

b). Desdobramentos das ações engendradas de acordo com o plano metodológico;

c). Realinhamento de escopo, ferramentas e cronologia, conforme entendimentos dos Grupos de Ação e de Suporte;

d). Concretização dos levantamentos de campo, de acordo com uma agenda negociada, contemplando pontos da metodologia e a partir de solicitações da fiscalização do contrato;

e). Execução de Projeto de Arquitetura, fundamentado na modelagem em ergonomia de concepção, derivada do método SPM ${ }^{\circledR}$.

Os registros das observações, bem como ações correspondentes ensejadas pela equipe de pesquisadores (ergonomistas), foram tratados pela equipe de suporte responsável pelo programa de iniciação científica, conforme esclarecido neste relatório. Para facilitar a compreensão do texto e de outros documentos gerados no contexto desse PIC, torna-se necessário apresentar algumas definições básicas das terminologias utilizadas (VIDAL, 2008; IIDA, 2005) .

Anotações de Projeto - Todo dado ou informação relevante obtido no transcorrer da Análise Ergonômica que se configura em um possível elemento do Projeto de Arquitetura, de forma a corresponder às necessidades de um sistema de trabalho.

Demanda (Demanda Ergonômica) - Toda solicitação que parte do cliente e para qual se espera uma ação correspondente da equipe de ergonomia. A Demanda Gerencial, portanto, confunde-se com o próprio escopo contratual.

Ferramentas de Escopo - São instrumentos de projeto ergonômico que fazem parte do conjunto de ferramentas de uso recorrente em ações do gênero.

Ferramentas de Gestão - São instrumentos da Gestão de Projetos definidos pela ação conjunta do Grupo de Ação Ergonômica e do Grupo de Suporte Ergonômico, específicos para utilização no projeto contratado. Podem ser de uso corrente, adaptados ou desenvolvidos especialmente para o projeto.

Grupo de Ação Ergonômica - Trata-se da equipe da empresa responsável pela condução, definição de objetivos e aprovação dos resultados dos trabalhos de campo desenvolvidos pelo Grupo de Suporte Ergonômico. 
Grupo de Suporte Ergonômico - Trata-se da equipe de ergonomistas atuante em campo, responsável pela aplicação do plano metodológico, composto da coleta de dados, tratamento e validação dos mesmos, auxílio ao diagnóstico e confecção dos relatórios preliminares de projeto.

Grupo de Gestão do Projeto - Trata-se da equipe responsável pela condução do Projeto Ergonômico e é composto pelos profissionais do Grupo de Suporte Ergonômico e do Grupo de Ação Ergonômica.

Método SPM $®$ - Método de Ação Ergonômica desenvolvido pelo Laboratório GENTE/COPPE/ UFRJ, que busca ampliar o alcance da ação ergonômica, utilizando-se de um conjunto de ações sistematizadas e uso de ferramentas de projeto participativo.

Modelo FOCA $®$ - Abordagem de Gestão de Projetos em Ergonomia, que busca a integração das dimensões Física, Organizacional e Cognitiva da Ergonomia em um projeto de Ambiente (o " $\mathrm{A}$ " do FOCA) de forma a equilibrar as necessidades de enquadramento normativo e conceitual dos sistemas de trabalho às necessidades dos usuários.

Modelo Operante - Um esquema definido a partir do mecanismo de interação e iteração entre os agentes de um processo de trabalho, que conduz ao estabelecimento de práticas e procedimentos e à compreensão da atividade de trabalho.

Modo Operatório - A maneira pela qual o trabalhador estabelece sua ação sobre os meios e sistemas de trabalho, respondendo às exigências da tarefa em contraponto com as regulações que se fazem necessárias, de modo a alcançar o resultado final desejado.

Organização do Trabalho - Sistema integrado de ações de respectivos indivíduos e grupos, contextos sociais e espaciais, além dos recursos envolvidos na operacionalização de um sistema produtivo.

Pré-diagnóstico - Diagnóstico preliminar que antecede a construção de um Modelo Operante de uma Situação de Trabalho. À medida que compreendemos a atividade, chegamos a um pré-diagnóstico.

Regulações - São respostas dos trabalhadores às imposições da tarefa, de modo a equilibrar o andamento de suas atividades de trabalho, compensando (por antecipação, mitigação, preparação, etc.) as restrições diversas que ocorrem no decorrer dessas operações produtivas.

Situação de Trabalho - Trata-se de uma combinação singular de fatores internos (estado orgânico, competência, personalidade) e externos (normas, meios de trabalho, equipamentos, etc.) num dado contexto de produção.

Sistema de Trabalho - Conjunto de procedimentos, equipamentos, dispositivos, recursos humanos e outros, que se constituem no interior de um Ambiente de Trabalho, sendo a razão de ser de toda a operacionalização desse conjunto. Em geral, entende-se um sistema de trabalho como um Sistema Produtivo.

\section{FUNDAMENTAÇÃO TEÓRICA}

No projeto de salas de controle integradas, devemos partir imediatamente da análise dos requisitos dos usuários do sistema. Isso difere de modelos de ação projetual convencionais, cujo encaminhamento básico é a composição arquitetônica, ainda que em ambientes de trabalho o normal seja que - em linguagem de design - a "forma siga a função". A atividade de controle pressupõe uma forte 
carga cognitiva com picos de atenção e posicionamento estático. Isso remete de imediato a questões de acústica, iluminamento, conforto estático, dentre outras.

De imediato podemos antecipar indicadores relacionados aos aspectos técnicos operacionais principalmente aqueles relacionados às questões normativas e regulatórias - mas também devemos considerar a utilização de "parâmetros sociais", ou seja, critérios que privilegiem a percepção sensorial em sua conexão com os aspectos cognitivos da atividade de controle. Controlar define-se, de acordo com as normas nacionais e internacionais, como um "conceito geral que denota influência proposital sobre algum objeto sendo monitorado". Esquematicamente, podemos representar os principais elementos de um sistema de controle, conforme a Figura 1.

Figura 1 - Modelo do processo de controle.

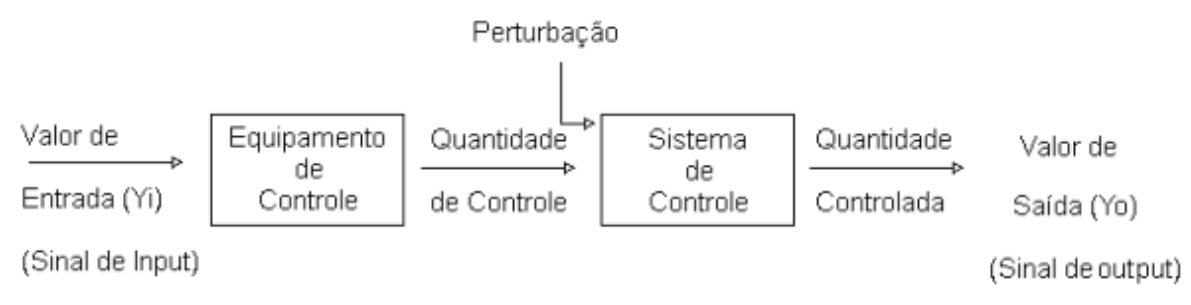

Fonte: (IVERGARD, 2008).

Se um dado sistema tem uma quantidade determinada de input e output, a relação entre estas é determinada por uma regra ou "função de transferência" (ou transformação) que depende de certo número de parâmetros (ps). Assim, a função de transferência de um sistema pode ser expressa pela equação:

$$
\text { Yo }=f(y: p s)
$$

Um sistema pode ser elucidado se as entradas, a função de transferência e as saídas são conhecidas (ISO 11064, 2004). Quando um fator - ou mais de um - não é conhecido, vários métodos podem ser usados para defini-los. As seguintes combinações de quantidades conhecidas e não conhecidas podem ocorrer:

1. Entradas, função de transferência e parâmetros são conhecidos e as saídas são requeridas, como no caso de um sistema em processo de design;

2. As saídas, funções e parâmetros são conhecidos e as entradas requeridas. O método usado nesse caso é o diagnóstico, utilizado para se descobrir o que se está errado a partir de um conjunto de "sintomas";

3. As entradas, saídas e função de transferência são conhecidas e os parâmetros são exigidos. Esse é conhecido como método de identificação, sendo utilizado quando se deseja uma descrição matemática de um dado evento.

4. As entradas e saídas são conhecidas e tanto a função de transferência como os parâmetros são exigidos. 0 método para esse tipo de problema é conhecido como "técnica da caixa 
preta" (black box) e é normalmente utilizado na descrição e testagem de sistemas técnicos complexos.

Em sistemas de controle de indústrias de processo, todas as quantidades são mais ou menos conhecidas, dependendo do nível de "identidade" do sistema, tal qual a opção 3. Um operador bem treinado e qualificado, assim como um sistema automatizado de controle, "reconhece" bem os vários parâmetros. Na prática, existem sempre fatores desconhecidos - perturbação (Yn) - que devem ser compensados por ações do operador e respostas desses sistemas de controle. No caso da operação de painéis de controle em indústrias de processo contínuo, devemos buscar entender como interagir e controlar as interfaces do sistema homem-máquina. Vários estudiosos têm colaborado na tentativa de se reproduzir, simular a atuação dos operadores como "processadores de informações". (IVERGARD, 2008).

O maior problema desses modelos é que eles, geralmente, lidam com somente uma variável do comportamento humano, o que torna a validade de aplicação desses modelos bastante limitada, até porque somente o comportamento observável pode ser auferido. Mesmo a tentativa de simulação de processos encontra obstáculos na sua utilização, já que estas são baseadas em representações de uma realidade e não da realidade em si. Porém, é possível gerar modelos que ajudem a descrever o comportamento humano frente a uma operação de um dado sistema a partir de uma modelagem sobre as capacidades de processamento e os processos cognitivos associados às mesmas. É aí que reside a importância de se definir os chamados "padrões de projeto" que nada mais são do que modelos de representação aceitáveis para um determinado contexto sociotécnico. Deve-se esclarecer que a SIC® não é um método, mas sim uma ferramenta metodológica desenvolvida para diferentes metodologias de ação ergonômica. No contexto do estudo em tela, ela se insere no processo de uma Análise Ergonômica de Concepção, voltada para incorporação de anotações de projeto em um processo de reconfiguração de uma sala de controle de uma refinaria de petróleo. Ela foi desenvolvida pelo Laboratório GENTE, vinculado à COPPE/UFRJ, para ser aplicada em situações em que haja necessidade de se eleger prioridades de ação em contextos com limitações de tempo ou escopo. Em sua forma mais geral, a ferramenta possui as seguintes características:

- Objetivo: Permitir uma priorização de ações ergonômicas fundamentada em parâmetros de semiquantitativos de alta e baixa amplitude, de modo a auxiliar no processo de gestão, sobretudo no que tange aos mecanismos de decisão.

- Justificativa: Ferramentas como o GUT, por exemplo, resultam em valores que são exclusivamente dependentes das impressões de um analista. A tabulação SIC permite que outros stakeholders de um projeto ou programa de ergonomia possam colaborar no estabelecimento de um índice de priorização de ações. Isso confere mais legitimidade e confiabilidade ao processo.

- Execução: Inicialmente, deve-se buscar a determinação de uma apuração GUT, que pode ser executada a partir das variáveis da análise (aspectos, impactos, ações corretivas, recomendações, etc). $O$ instrumento é bastante versátil, podendo ser ajustado de acordo com variáveis que sejam coerentes e relevantes ao projeto em foco, sempre validadas pela força de trabalho. Após a apuração GUT, constrói-se a matriz de criticidade para cada atividade específica.

A matriz SIC gerada a partir da tabulação dos indicadores de severidade se qualifica como instrumento de avaliação coerente com a natureza de subjetividade inerente à ação projetual para salas de controle - e para outras formas de ação projetual também. Ao utilizar-se de dados resultantes de medidas semi-quantitativas (ou "quali-quantitativas") ela apropria elementos fundamentais para a 
condução de projeto que passariam imperceptíveis por processos de avaliação puramente quantitativos e determinísticos.

Entretanto, na prática, como podemos nos beneficiar da ação ergonômica e obter resultados satisfatórios na solução de projetos dessa natureza? Compreendendo a ergonomia como função de conformidade operacional para sistemas de trabalho, podemos atribuir as suas três dimensões aceitas - física, cognitiva e organizacional - uma correspondência direta aos usuários, ao seu ambiente de trabalho e ao próprio sistema de trabalho. Assim, do ponto de vista da ergonomia física, um painel de controle de grandes dimensões permite uma menor sobrecarga do sistema visual, redução de movimentos de pescoço e melhor postura, com redução de movimentos. A questão da antropometria também se torna menos importante, haja vista que telas grandes podem ser visualizadas de certa distância, evitando movimentos de vai e vem com o conjunto operador-cadeira.

Em termos da ergonomia cognitiva, a utilização de telas maiores permite o reconhecimento de um conjunto de indicadores e variáveis simultaneamente, facilitando o reconhecimento das possíveis circunstâncias da operação e o processo decisório. Por último a questão da ergonomia organizacional também é otimizada pelo fato de que grandes telas podem ser visualizadas confortavelmente por um grupo maior de pessoas em overview (plano amplo). Como subproduto, a utilização da tecnologia pode possibilitar a melhoria da produtividade e qualidade de operação a partir de uma melhor interação coletiva e colaborativa na atividade de trabalho. Existe uma lista de verificação desenvolvida por pesquisadores dinamarqueses (THOMPSON et al., 2003) que foi desenvolvida para o projeto torres de controle em embarcações, cujas características do processo são compatíveis com aquelas dos centro de controle integrado. Esses pontos de investigação são úteis em combinação com outras listas de verificação ergonômica e estão resumidos no Quadro 1.

\section{Quadro 1 - Lista de Verificação de atividades de controle}

\begin{tabular}{|l|l|}
\hline Riscos & Investigar \\
\hline Ruidos / Vibrações & $\begin{array}{l}\text { Sons ou vibrações que possam causar danos ao sistema auditivo ou } \\
\text { ao organismo como um todo. }\end{array}$ \\
\hline Toxidades & $\begin{array}{l}\text { Gases ou contaminantes presentes no ambiente ou de origem } \\
\text { externa que possam causar envenenamento ou doenças de } \\
\text { desenvolvimento gradual }\end{array}$ \\
\hline Elétricos & $\begin{array}{l}\text { Equipamentos que possam provocar choques por contato ou } \\
\text { irradiação. }\end{array}$ \\
\hline Mecânicos & $\begin{array}{l}\text { Equipamentos com partes móveis que possam causar danos } \\
\text { diversos. }\end{array}$ \\
\hline Músculo-esqueléticos & $\begin{array}{l}\text { Tarefas que afetam adversamente a musculatura ou o sistema } \\
\text { esquelético separadamente ou de forma combinada. }\end{array}$ \\
\hline Térmicos & $\begin{array}{l}\text { Fontes de geração com niveis incompativeis com o conforto ou } \\
\text { segurança de operação. }\end{array}$ \\
\hline Oticos & Equipamentos capazes de provocar danos ao sistema ocular. \\
\hline Radiações Eletromagnéticas & $\begin{array}{l}\text { Outras fontes de energia: Ex: Campos Magnéticos, Microondas, } \\
\text { etc. }\end{array}$ \\
\hline
\end{tabular}

Fonte: (THOMPSON et al. , 2003) 


\section{DESCRIÇÃO DAS ATIVIDADES}

A partir dos levantamentos realizados pelos ergonomistas, as primeiras demandas de tratamento de dados foram relacionadas à percepção do espaço de trabalho, que vai além do conceito de Ambiente de Trabalho, pois combina a noção de ambiente (e ambiência) com tempo e movimento. Alguns arquitetos chamam essa relação tempo-movimento de "quarta dimensão" da arquitetura. De uma forma simples, a raiz do questionamento remete à ideia de que um dado ponto observado e focalizado possui, além de suas três dimensões básicas, um estímulo sensorial que se transforma a partir da mudança de posição - e do tempo correspondente ao deslocamento - do observador.

De forma a situar melhor a questão, o grupo de suporte realizou uma série de entrevistas e aplicação de roteiros direcionados - uma forma de questionário mais dinâmico - objetivando avaliar as percepções dos operadores em relação ao seu espaço e à própria organização do trabalho. Isso é fundamental para a formulação de uma orientação básica dos encaminhamentos da ação projetual, já que fornecem indicações dos aspectos prioritários, de acordo com a percepção dos operadores. Combinando-se esses aspectos com as orientações técnicas e normativas relacionadas à operacionalização do sistema de trabalho, encontramos o ponto ótimo para as soluções de projeto.

Esses dados preliminares, obtidos no decorrer da ação ergonômica, transformam-se em anotações de projeto, sendo, portanto, informações relevantes para a tradução das expectativas dos usuários em ações projetuais, resolvendo não apenas questões relacionadas às condições de trabalho como na qualidade - e produtividade - do mesmo. 0 resultado da tabulação, convertido em gráficos de sensações, será apresentado a seguir bem como as tabulações dos indicadores de projeto e a matriz de criticidade.

Uma vez concluída a atividade de campo, os pesquisadores do Laboratório GENTE/COPPE/UFRJ solicitaram ao Professor responsável pelo PIC, também vinculado ao laboratório, que coordenasse, no contexto do programa em questão, as ações de tratamento de alguns dos dados coletados, sobretudo aqueles relacionados aos questionários e check-lists que visavam à determinação dos níveis de satisfação da força de trabalho. Esses instrumentos são componentes essenciais da ferramenta SIC®, que corresponde a uma matriz de tabulação de dados de severidade (ou criticidade), possibilitando, pela eleição de ações de priorização, o encaminhamento decisório em geral. No nosso caso, o SIC foi apropriado para a função de ferramenta de gestão de projetos, ou seja, as inferências definidas pelas matrizes estabelecem pontos de anotação de projeto. Cabe ressaltar que o processo de gestão aqui referido é especificamente relacionado ao projeto de ambientes de trabalho.

\section{TRATAMENTO DOS DADOS}

Ficou definido que, por conta de restrições contratuais e de segurança das informações, bem como para evitar eventuais implicações éticas perante o UniFOA, a equipe do PIC ficou responsável apenas por: tabulação dos gráficos de tendência, apresentados a seguir; resumo das anotações de enquadramento normativo e das fichas de emprego de tempo (ainda em curso); pesquisa documental necessária à construção dos IDPs - Indicadores de desempenho de projeto.

Uma vez resolvida a questão do encaminhamento da ação projetual, o passo seguinte foi a determinação dos indicadores de criticidade, que não mais ficou diretamente a cargo da equipe do PIC, responsável apenas pela tabulação dos dados de acordo com as fórmulas apresentadas pela coordenação do projeto. A partir da definição dessas variáveis - os indicadores de gravidade ocupacional - foi possível então priorizar as ações relativas à gestão de projetos, de acordo com uma escala crescente e decrescente, denominada Tabulação "SIC" ® (Somatório dos Índices de Criticidade). 


\subsection{Elaboração dos Gráficos de Tendência}

O levantamento de sensações decorrente dos estudos de campo evoluiu para um tratamento mais direcionado à ação projetual. De forma a permitir uma visualização quase imediata das preferências de cada operador e do coletivo de trabalhadores, foram construídos os chamados "Gráficos de Tendência" que combinam o nível de satisfação ou outras prioridades de escolha que devem ser tomadas para o sequenciamento das atividades de projeto.

Como poderemos observar, a linha vermelha segue a opção de cada perfil de trabalhador. A ordem da opinião de cada operador não é tão importante, mas deve-se seguir a sequência de opiniões individualizadas para todas as questões colocadas. Em outras palavras, o mesmo respondente do primeiro quesito é o primeiro a opinar em relação ao segundo aspecto e todos os demais em uma mesma sequência. A seguir é apresentado um dos gráficos resultantes das respectivas tabulações em modelagem Likert. A adoção do modelo já fornece uma visão inicial da tendência associada ao quesito analisado, de acordo com a visão dos usuários diretos do sistema. 
Quadro 2 - Exemplo de Gráfico de Tendência

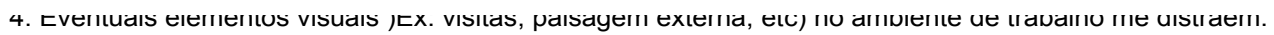

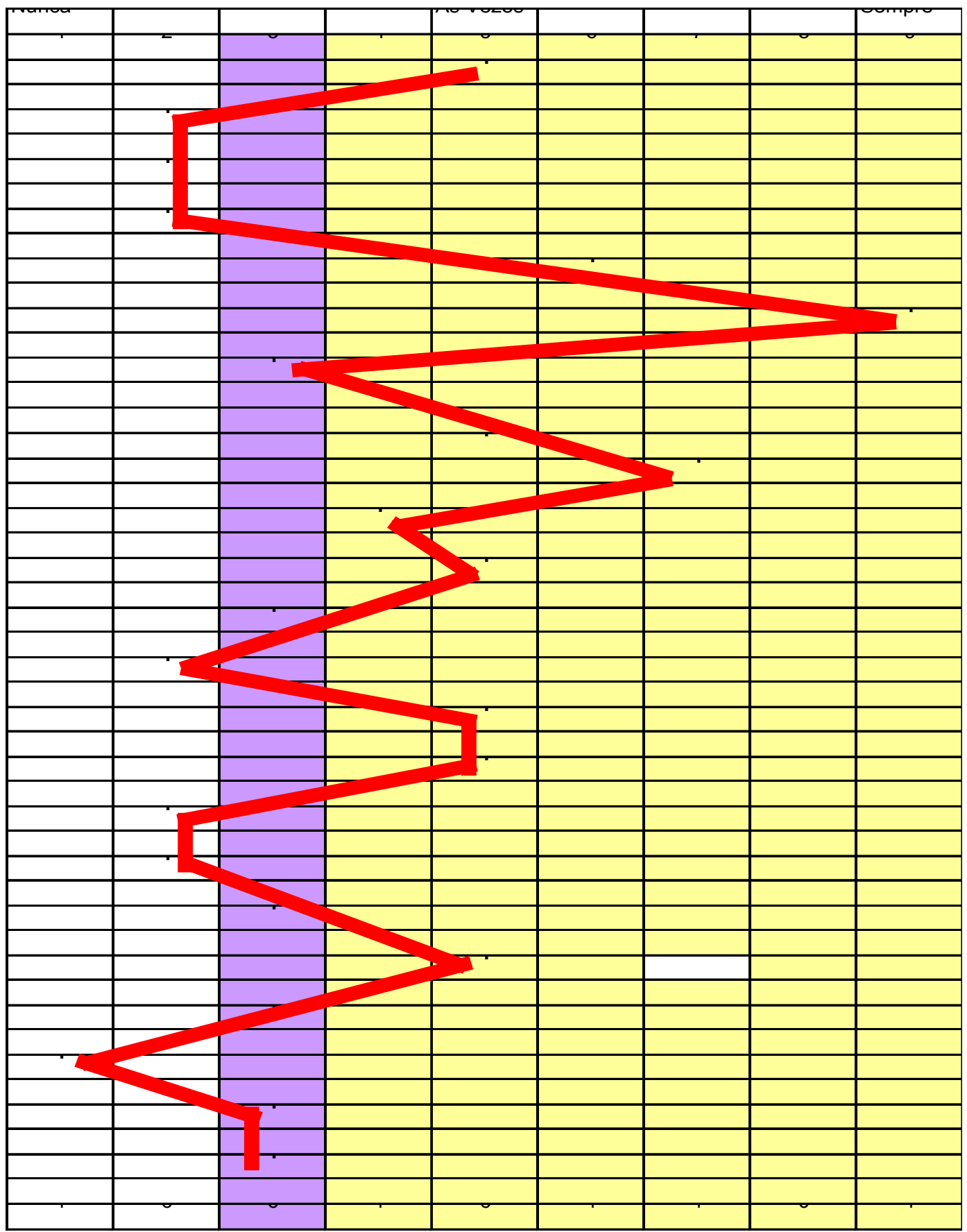

\subsection{Tabulação dos Indicadores SIC}

O acrônimo SIC significa "Somatório dos Indicadores de Criticidade" e é uma ferramenta de avaliação macro desenvolvida pelo Laboratório GENTE/COPPE/UFRJ. Os indicadores de criticidade são medidas quali-quantitativas das condições de trabalho em uma organização. No seu emprego mais geral, eles objetivam identificar prioridades de ação ou determinar marcos decisórios com base em uma "coerência sociotécnica". No modelo desenvolvido para a empresa em foco, o SIC entrou na composição 
das variáveis a serem empregadas na fórmula de cálculo do tempo de reposição de fadiga, uma vez que uma situação ergonomicamente bem agenciada é suscetível de reduzir a fadiga de forma contundente.

Para obtermos o Índice SIC, utilizamos os resultados da apreciação ergonômica, realizados por ergonomistas, que nos permitiram extrair os valores de cada situação de trabalho. Isso é feito ponderando-se os dados levantados de acordo com a caracterização de cada atividade. A tabulação SIC determina o grau de risco ergonômico de cada posto de trabalho, portanto a partir da combinação de variáveis de diferentes matrizes (Matriz Observacional, Enquadramento Normativo e demais ferramentas de apreciação e de avaliação global das situações de trabalho). O resultado da tabulação SIC nos é dado por:

\section{$\mathrm{SIC}=[\text { Fator Criticidade }]^{*}[\text { Apuração GUT }]^{*}[$ Numero de desconformidades $]$}

O fator de criticidade, assim como o quantitativo de desconformidades, é resultado direto da avaliação ergonômica. A Matriz de GUT deriva de uma ferramenta de priorização que apropria e quantifica o nível de importância de tratamento de cada posto de trabalho. A avaliação final é dada pela fórmula que se segue:

$$
\mathrm{AV}_{\text {Situação }}=\Sigma_{\mathrm{j}=1, \mathrm{n}}\left[\mathrm{G}_{\mathrm{i}}^{*} \mathrm{U}_{\mathrm{i}} * \mathrm{~T}_{\mathrm{i}}\right]
$$

As matrizes já consolidadas, cujo exemplo é apresentado a seguir, funcionaram no caso da ação projetual como suporte decisório para a escolha de critérios de projeto na reformulação não apenas da sala de controle, mas de todo o Centro de Controle Integrado (CCl).

Quadro 3 - Exemplo de Tabulação SIC para um dos postos de trabalho no $\mathrm{CCI}$.

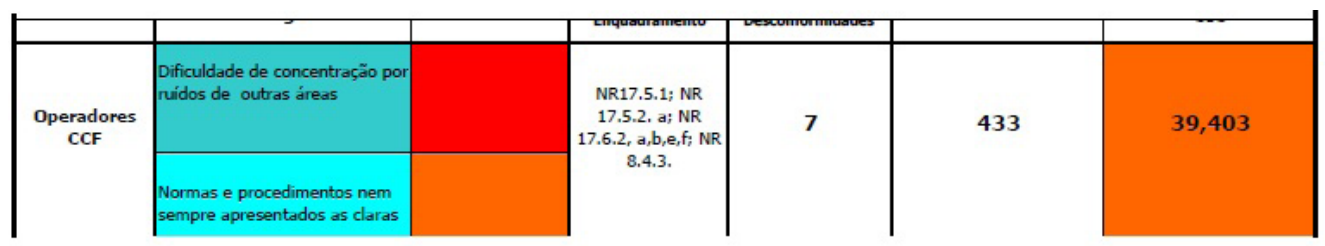

\begin{tabular}{|c|c|c|c|c|c|c|}
\hline \multicolumn{3}{|c|}{ Variáveis de Risco } & G & $\mathrm{U}$ & $\mathbf{T}$ & Apuração local \\
\hline \multirow{2}{*}{\multicolumn{2}{|c|}{ Sobrecargas }} & Fisica & 2 & 2 & 2 & 8 \\
\hline & & Mental & 3 & 3 & 2 & 18 \\
\hline \multirow[t]{3}{*}{ Ambiente } & Dimensionamento & & 5 & 5 & 2 & 50 \\
\hline & Ambiência & & 4 & 3 & 3 & 36 \\
\hline & Acessos & & 4 & 3 & 2 & 24 \\
\hline \multirow[t]{4}{*}{ Equipamentos } & Bancadas e/ou mesas & & 1 & 1 & 1 & 1 \\
\hline & EPI & & 1 & 1 & 1 & 1 \\
\hline & Maquinário & & 1 & 1 & 1 & 1 \\
\hline & Dispositivos de Controle & & 1 & 1 & 1 & 1 \\
\hline \multirow[t]{3}{*}{\begin{tabular}{|l|}
$\begin{array}{l}\text { Orga nização do } \\
\text { Trabalho }\end{array}$ \\
\end{tabular}} & Normas de produção & & 4 & 4 & 4 & 64 \\
\hline & Exigência de tempo & & 4 & 4 & 3 & 48 \\
\hline & Conteúdo da tarefa & & 4 & 5 & 5 & 100 \\
\hline
\end{tabular}




\section{CONCLUSÕES E ENCAMINHAMENTOS}

0 projeto de arquitetura resultante das ações ergonômicas, gerado de forma integrativa e participativa pela equipe de ergonomia e os usuários do sistema, ainda não foi executado pela empresa que contratou a avaliação ergonômica. Entenda-se projeto como o detalhamento executivo arquitetônico para construção, pois o resultado global, que inclui o relatório de apreciação ergonômica e o programa de necessidades, foi entregue e aceito pelo contratante. Esse relatório final incorporou os resultados das ações desenvolvidas no contexto do Projeto de Iniciação Científica desenvolvido pelos autores, o que atesta o reconhecimento das partes em relação à qualidade da pesquisa desenvolvida.

Com relação ao uso da ferramenta "Tabulação SIC", esta se mostrou bastante eficaz ao direcionar as ações de priorização e decisão para o projeto. A matriz funcionou como uma espécie de plano de ação de ergonomia de concepção, permitindo a adoção de critérios por vezes conflitantes entre si, sobretudo em aspectos que colocam em um plano decisório deferentes escolhas, como por exemplo, entre adotar um equipamento de segurança e outro que permita maior conforto aos operadores. Portanto, a ferramenta fornece argumentações adicionais que transformam as decisões críticas de projeto numa démarche de convergência salutar para a operacionalização de ambientes construídos, sobretudo aqueles de natureza complexa. Uma das constatações pela análise das matrizes foi que os operadores das estações de tratamento de água e esgotos eram aqueles mais diretamente afetados por inadequações de ordem ocupacional, de conforto e de segurança, fato nunca antes identificado, devido à assunção que estes teriam algo mais preocupante para cuidar em suas próprias rotinas.

Como limitação do estudo, pode-se apontar que certas atitudes gerenciais na refinaria podem ter afetado de alguma forma as respostas de alguns usuários. Isso sugere um processo futuro de conscientização e foco na pesquisa de campo que possa mitigar ou mesmo eliminar esses efeitos. Entretanto, essa situação não afetou as ações do projeto de iniciação científica, já que não houve contato direto entre os envolvidos no projeto e o corpo funcional da refinaria onde o estudo foi realizado. De fato, experiências prévias em outros projetos semelhantes que utilizaram a ferramenta mostram que sempre haverá uma dose de subjetividade presente na análise observacional, o que não é exclusividade da ferramenta SIC e sim de qualquer modelo de priorização de decisões (VIDAL, 2013).

O cliente fim, ou seja, o Laboratório GENTE/COPPE/UFRJ, que acolheu e deu suporte à equipe do PIC, confirmou que foram cumpridos os objetivos do programa, já que o resultado prático - o projeto de arquitetura final, integral e consensual - foi devidamente aceito sem ressalvas. Isso atesta a validade das ações e a qualidade dos métodos e ferramentas utilizados. De fato, a ferramenta SIC continua sendo utilizada por ergonomistas em todo o país (MASCULO, 2013), principalmente em função de seu caráter flexível de aplicação e abrangência, sem afastar-se do viés científico de sua proposta original. 


\section{REFERÊNCIAS}

ÄSTRAND, Per \& ROHDAL Kaare. Tratado de fisiologia do esforço. Rio de Janeiro: Interamericana, 1984.

BRASIL. Manual de aplicação da Norma Regulamentadora 17. $2^{\text {a }}$ ed. Brasília: MTE , SIT , 2002

BRASIL. Ministério do Trabalho e Emprego, Norma Regulamentadora 15. Brasília: MTE 2000.

GRANDJEAN, Etienne. Manual de ergonomia: adaptando o trabalho ao homem. 4. ed. Porto Alegre: Bookman, 1998.

IIDA, Itiro. Ergonomia: projeto e produção. 2. Ed. São Paulo: Ed. Edgard Blucher, 2005.

IVENGARD,Toni; HUNT, Brian. Handbook of control room design and ergonomics. London: CRC Press, 2008.

INTERNATIONAL ORGANIZATION FOR STANDARDIZATION. ISO 3382: Acoustics- Measurement of the reverberation time of rooms with reference to other acoustical parameters.

ISO 9241: series ISO Ergonomics of human-system interaction - Part 110: Dialogue Principles.

ISO 11064:2004: Ergonomic design of control centers - Part 1 - Principles for the design of control;

Part 2 - Principles for the arrangement of control suites; Part 3 - Control room layout; Part 4 - Layout and dimensions of workstations; Part 5- Displays and controls; Part 6 - environmental requirements of control centers; Part 7; Principles for the evaluation of control centers.

LABORATÓRIO GENTE. Relatório Final de Projeto "Projeto Ergonômico de Reformulação do CCI", Rio de Janeiro: COPPE/UFRJ, 2010.

LABORATÓRIO GENTE. Relatório Final de Projeto "RFad - Avaliação Técnica do Tempo de Reposição de Fadiga - Fundamento e Modelo". Rio de Janeiro: COPPE/UFRJ, 2010.

MÁSCULO, Francisco; COLAÇO, Geraldo. Ergonomia na Indústria Calçadista: case apresentado no XXXIII Encontro Nacional de Engenharia de Produção - ENEGEP, 2013.

SANDERS, Mark; McCORMICK, Ernest. Human Factors in engineering and design. 7th ed, 1993.

SILVA E SANTOS, Marcello. Arquitetura de Locais de Trabalho. In: Ergonomia: trabalho adequado e eficiente. São Paulo: Editora Elsevier, 2011.

THOMPSON. L.K.. Facing Diversity when designing and evaluating driver support systems, in Meeting Diversity in Ergonomics. Oxford: Elsevier, (Pikaar, Ruud et. al. eds.), p. 289-309, 2007.

VIDAL, Mario Cesar. Guia para análise ergonômica do trabalho na empresa: uma metodologia, realista, ordenada e sistematizada. Rio de Janeiro: $3^{\mathrm{a}}$ ed. Editora Virtual Científica, 2008.

Comunicação pessoal, 2013. 